\title{
Visibility Computations: From Discrete Algorithms to Real Algebraic Geometry
}

\author{
Thorsten Theobald
}

\begin{abstract}
We investigate visibility computations with moving viewpoints. The initial problems are of discrete and algorithmic nature, but even for simple classes of objects (such as balls and polytopes), they lead to interesting and difficult problems from real algebraic geometry. Namely, it is necessary to characterize and compute the common tangent lines to a given set of convex bodies.

In particular, we present a new sweep algorithm in dimension 2, as well as survey and extend recent algebraic-geometric results on the tangent problems in dimension 3 .
\end{abstract}

\section{Introduction}

Visibility computations belong to the central tasks in computer graphics and computational geometry [19]. Depending on the particular framework and on the particular question to be answered, there is a large variety of mathematical and algorithmic core problems behind these questions.

In the simplest case of a visibility problem, we are given a fixed viewpoint $v \in \mathbb{R}^{n}$ (say, $n \in\{2,3\}$ ), and the scene consists of a set $\mathcal{B}$ of bodies. The task is to compute a suitable two-dimensional projection of the scene ("to render the scene") that reflects which part of the scene is visible from the viewpoint $v$. In a more dynamic and interactive setting, the viewpoint can be moved interactively (see, e.g., $[\mathbf{4}, \mathbf{1 5}])$. However, in general, after each movement of the viewpoint a new rendering process is necessary. In order to speed up this process, commercial renderers apply caching techniques [34].

From the algorithmic and geometric point of view it is desirable to establish a more global view of the scene in advance and answer questions like: Which of these bodies can be partially seen from some viewpoint within a given viewpoint area? Those bodies which are not visible from any of these viewpoints can be removed from the whole visualization process in advance. In case of dense crystals whose atoms are visualized as sufficiently large balls, this can reduce the time consumption of the rendering process significantly (see Fig. 1).

Key words and phrases. visibility, tangent lines, spheres, transversals, enumerative geometry, real algebraic geometry, computational geometry.

2000 Mathematics Subject Classification. Primary 26C10, 68U05; Secondary 14N10, 51M30. 


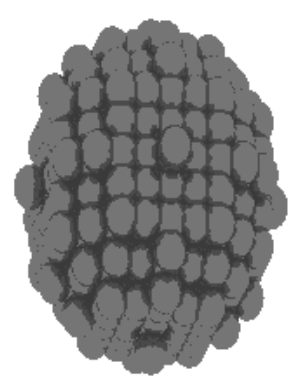

Figure 1. Dense arrangement of atoms

So far, there exists only few literature on visibility questions with moving viewpoints in dimension at least three (see $[4, \mathbf{1 5}, \mathbf{3 3}]$ ). A major reason for this can be seen by various intrinsic difficulties in the underlying geometric questions.

From the algebraic-geometric point of view, visibility computations with moving viewpoints require to study the interaction of the geometric bodies with lines. In particular, it is essential to characterize certain extreme situations which correspond to common tangent lines to a given number of bodies. In dimension 2 , the resulting geometric questions typically remain rather elementary, and the main focus is on the algorithmic side. In contrast to this, in dimension 3, even for simple types of bodies, such as spheres, the underlying geometric problems have a high algebraic degree and hence give rise to interesting and difficult questions of real algebraic geometry. Several recent papers deal with these algebraic-geometric core problems $[\mathbf{1 7}, \mathbf{1 8}$, 31, 29, 32]. In particular, the relevant real enumerative aspects constitute a rich and natural class of geometric examples within the general framework of real enumerative geometry (see the survey $[\mathbf{2 8}]$ ).

In the present paper, we want to explore this range between algorithmic and real algebraic-geometric issues by exemplifying several corresponding aspects. We proceed along the following outline. In Section 2, the necessary notation is introduced. Then, in Section 3, we discuss algorithmic aspects of the visibility problems and present a new sweep algorithm in $\mathbb{R}^{2}$. Section 4 surveys recent results on the algebraic-geometric tangent problems in $\mathbb{R}^{3}$. By discussing an optimization aspect of the tangent problems in Section 5, we illustrate some of the proof techniques for the tangent results, and also establish a connection to the optimization of polynomial functions over the real numbers.

\section{Notation}

As an example for the applications mentioned in the introduction, consider the following problem from ray-tracing with moving viewpoints. Here, we want to compute information on the viewpoint positions where the visibility topology of the scene changes. As a special case, this includes tackling the following core problem of partial visibility.

A set $B \subset \mathbb{R}^{n}$ (say, $n \in\{2,3\}$ ) is called a (convex) body if it is bounded, closed, convex, and contains an interior point. Now we consider a scene consisting of a set $\mathcal{B}$ of (not necessarily disjoint) bodies from a specific class $\mathcal{X}$ in $\mathbb{R}^{n}(\mathcal{X}$ might be the set of all balls or the set of all polytopes). A body $B \in \mathcal{B}$ is called partially 
visible from a viewpoint $v$ if there exists a line segment connecting $v$ and $B$ not intersecting with the interior of any other body in $\mathcal{B}$. A body $B \in \mathcal{B}$ is called partially visible if $B$ can be seen from some viewpoint "outside" of the scene, i.e., if there exists a ray starting at $B$ not intersecting with the interior of any other body in $\mathcal{B}$. We call such a ray a visibility ray for $B$. Bodies which are not partially visible can be immediately removed from the scene, which reduces the complexity of the visualization process.

\section{A Sweep Algorithm for the Two-Dimensional Case}

We present efficient algorithms for solving the partial visibility problem in $\mathbb{R}^{2}$. Here, we are not only interested in checking partial visibility of one of the bodies but also in computing all bodies which are not partially visible. In order to avoid several special cases we assume that the bodies are pairwise disjoint.

Let $\mathcal{B}:=\left\{B_{1}, \ldots, B_{m}\right\}$ be a set of disjoint bodies in the plane. In the twodimensional case, checking partial visibility of a body $B \in \mathcal{B}$ can be reduced to a finite number of geometric problems as follows (cf. the treatment of stabbing lines in [11]). Without loss of generality let $|\mathcal{B}| \geq 2$ and assume $B=B_{1}$. If there exists a visibility ray for $B$ then we can continuously transform (i.e., rotate and translate) the visibility ray until we reach a situation where the underlying line is tangent to at least two of the bodies (one of them might be $B_{1}$ itself). Hence, it suffices to compute the set of all common tangent lines to a pair of bodies in $\mathcal{B}$ and check whether one of these lines contains a visibility ray. For any pair of disjoint bodies, the number of common tangent lines is exactly 4 (which can be seen as a very special case of the results in $[\mathbf{6}, \mathbf{1 6}]$ on the number of common supporting hyperplanes in general dimension).

In order to handle any class of bodies in the plane algorithmically, we have to assume that we can perform the following operations on this class.

(1) Compute the 4 common tangent lines to 2 bodies $B_{i}, B_{j}$.

(2) Compute the at most 2 intersections of a ray or a line with a body $B_{i}$.

In the following, we assume that we have access to two oracles performing these operations. Obviously, for the class of discs, the class of polygons, and the class combining discs and polygons these oracles can be realized quite easily. In particular, if the maximum number of vertices of any polygon is bounded by a constant then both oracles can be implemented in constant time.

DeFinition 1. A straight line is called critical if it is tangent to at least two bodies $B_{i}, B_{j}$ with $1 \leq i \neq j \leq m$. A ray is called critical if it is contained in a critical straight line.

Hence, the body $B_{1}$ is partially visible if and only if there exists a critical visibility ray for $B_{1}$. Consequently, it suffices to compute the set of critical lines and to check whether a critical line contains a visibility ray for $B_{1}$. Obviously, checking whether a given straight line contains a visibility ray for $B_{1}$ can be achieved with $O(m)$ calls to oracle 2 .

THEOREM 2. In dimension 2, the set of all partially visible bodies can be computed with $O\left(\mathrm{~m}^{3}\right)$ arithmetic steps, $O\left(\mathrm{~m}^{2}\right)$ calls to the first oracle and $O\left(\mathrm{~m}^{3}\right)$ calls to the second oracle. 


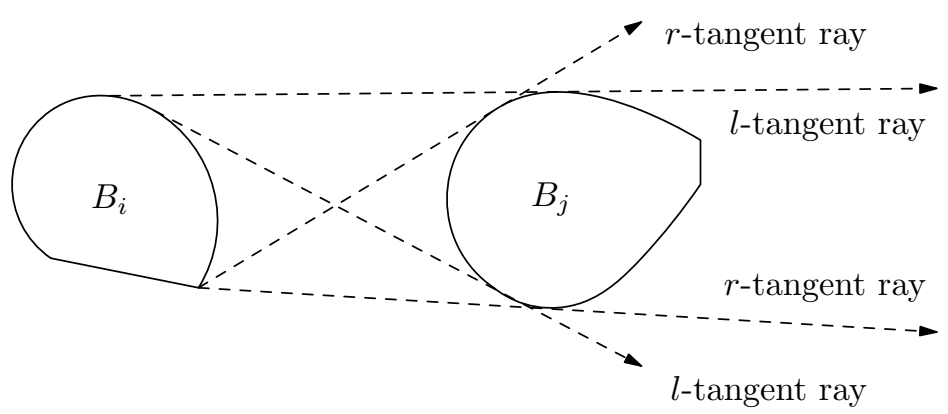

FiguRE 2. $r$ - and $l$-tangent rays from $B_{i}$ to $B_{j}$

Proof. There are $4 \cdot\left(\begin{array}{c}m \\ 2\end{array}\right)$ (not necessarily different) critical lines. For each critical line $l$ it can be computed with $O(m)$ arithmetic steps and $O(m)$ calls to the second oracle which bodies intersect with $l$ and which bodies are visible with regard to the line $l$.

The algorithm of Theorem 2 computes the set of all partially visible bodies in cubic time. However, the straighforward idea to modify it to a quadratic time algorithm for checking partial visibility of one specific body does not work. The reason is that it is a priori not clear which of the $O\left(\mathrm{~m}^{2}\right)$ critical lines can be omitted. If we are only interested in partial visibility of one specific body, say $B_{1}$, we can do better by using the following plane sweep algorithm requiring $O\left(m^{2} \log m\right)$ time and $O(m)$ space. (For extensive material on sweep techniques we refer to [9].)

We interpret the 4 common tangent lines of two bodies $B_{i}$ and $B_{j}$ as rays starting in some boundary point of $B_{i}$. As a consequence of the results in $[\mathbf{6}, \mathbf{1 6}]$, there are two tangent rays such that $B_{i}$ is on the left side of these tangent rays (" $r$-tangent rays of $B_{i}$ "); and there are two tangent rays such that $B_{i}$ is on the right side of the tangent rays ("l-tangent rays of $B_{i}$ "), see Figure 2.

For checking visibility of $B_{1}$ we first investigate the $2(m-1)$ (not necessarily different) $r$-tangent rays touching $B_{1}$ and some other body $B_{i}, 2 \leq i \leq m$. For each $r$-tangent ray we consider the outer normal $u \in \mathbb{S}^{1}$ where $\mathbb{S}^{1}$ denotes the unit sphere in $\mathbb{R}^{2}$; with each of these normals $u \in \mathbb{S}^{1}$ we associate the corresponding angle $0 \leq \alpha<2 \pi$ measured from the positive $x$-axis. As described in the following algorithm, we sweep the $r$-tangents according to increasing angles.

Subalgorithm for sweeping the $r$-tangents of $B_{1}$ and $B_{i}, 2 \leq i \leq m$ :

(1) Compute the set of $r$-tangents of $B_{1}$ and $B_{i}, 2 \leq i \leq m$, and sort them by increasing angles.

(2) Compute the number of intersections of the first $r$-tangent with bodies $B_{i}, i>1$.

(3) Consider the $r$-tangents successively in the order of increasing angles. In each of these steps do:

(a) Update the number of intersections with bodies $B_{i}, i>1$.

(b) If the number of intersections is 0 , then $B_{1}$ is partially visible; STOP.

For the update step we use the following lemma. 


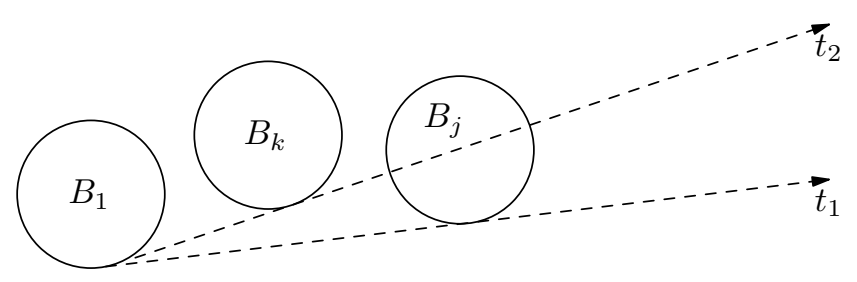

FiguRE 3. Update step during the sweep

LEMma 3. Let $t_{1}$ and $t_{2}$ be $r$-tangent rays of $B_{1}$ with angles $0 \leq \alpha_{1}<\alpha_{2}<2 \pi$, and let $C$ be some body with $t_{1} \cap C \neq \emptyset, t_{2} \cap C=\emptyset$. Then there exists an $r$-tangent ray to $B_{1}$ with angle $\alpha_{0} \in\left[\alpha_{1}, \alpha_{2}\right)$ which is tangent to $C$.

Proof. For any $\alpha \in[0,2 \pi)$ there exists some oriented tangent to $B_{1}$ with angle $\alpha$ (see, e.g., $[\mathbf{5}]$ ) and therefore some $r$-tangent ray to $B_{1}$ with angle $\alpha$. Let $\alpha_{0}$ be the supremum of $\alpha \in\left[\alpha_{1}, \alpha_{2}\right)$ such that the tangent with angle $\alpha$ intersects with $C$. Since $C$ is compact the tangent with angle $\alpha_{0}$ is tangent to $B_{1}$ and $C$, i.e., the supremum is a maximum.

In each step of the sweep we update the number of intersections of the sweep ray with bodies $B_{i}, i \geq 2$, in the following way. Let us first consider the case where the new angle $\alpha_{2}$ is strictly larger than the current angle $\alpha_{1}$ and where the $r$-tangent rays with angle $\alpha_{1}$ and $\alpha_{2}$ are each tangent to exactly two bodies. Let the $r$-tangent ray with angle $\alpha_{1}$ be tangent to $B_{1}$ and $B_{j}$, and let the $r$-rangent ray with angle $\alpha_{2}$ be tangent to $B_{1}$ and $B_{k}, 2 \leq j \neq k \leq m$. Then we only have to check whether the ray with angle $\alpha_{2}$ intersects with $B_{j}$ (i.e., if the sweep ray is just "entering" $B_{j}$ ) and if the ray with angle $\alpha_{1}$ intersects with $B_{k}$ (i.e., if the sweep ray is just "leaving" $B_{k}$ ); see Figure 3 . Due to Lemma 3 any additional change would imply the existence of some $r$-tangent with angle $\alpha_{0} \in\left(\alpha_{1}, \alpha_{2}\right)$. Consequently, the update step can be done in constant time. If there are several $r$-tangent rays with the same angle we can combine these update steps. The amortized costs for the update step are not larger than in the case of different angles. If during the sweep we reach a situation where the number of intersections is 0 then $B_{1}$ is partially visible and we can stop immediately. After the inspection of the $r$-tangent rays of $B_{1}$ the $l$-tangent rays of $B_{1}$ are swept in the same way.

So far, we have inspected the $r$ - and $l$-tangent rays of $B_{1}$. However, a visibility ray of $B_{1}$ is not necessarily tangent to $B_{1}$, and we also have to investigate the common tangents of bodies $B_{i}, B_{j}$ with $2 \leq i \neq j \leq m$. More precisely, for every fixed $i \in\{2, \ldots, n\}$, we consider the critical rays which are tangent to $B_{i}$. Here, we start the sweep with that $r$-tangent ray from $B_{i}$ to $B_{1}$ that has $B_{1}$ on the left side (see Figure 4 ). For this ray we count the number of intersections between $B_{i}$ and $B_{1}$, and separately we count the number of intersections of the backward ray with other bodies. Now we sweep the $r$-tangent rays of $B_{i}$ according to increasing angles and update the number of intersections between $B_{i}$ and $B_{1}$ as well as the number of intersections of the backward ray. If we reach a situation where both numbers of intersections are simultaneously zero then $B_{1}$ is partially visible and we can stop immediately. In any case, the algorithm can stop if the $r$-tangent ray to $B_{i}$ has $B_{1}$ 


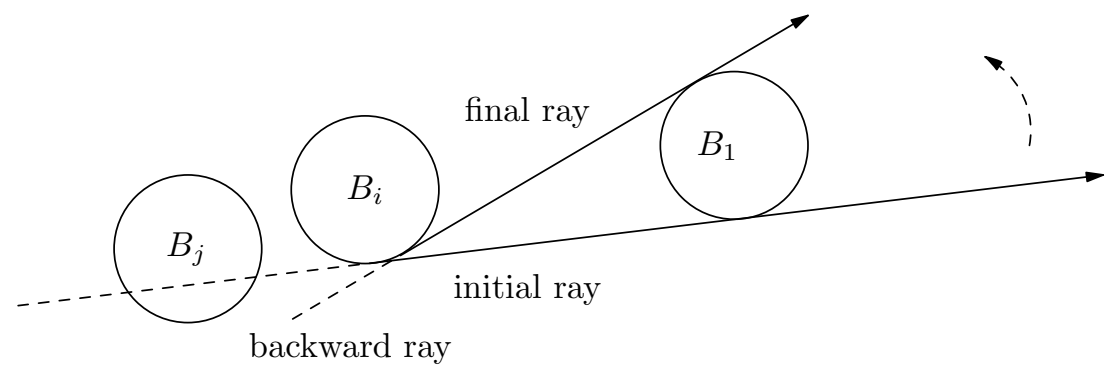

FIGURE 4. Initial and final ray for sweeping the $r$-tangent rays of $B_{i}, i \geq 2$

on its right side; see the illustration in Figure 4. After sweeping the $l$-tangent rays of $B_{i}$ the $r$-tangent rays of $B_{i}$ are investigated in the same way.

The correctness of the whole algorithm follows from the fact that the sweep inspects all $O\left(\mathrm{~m}^{2}\right)$ critical visibility lines and that the update step is correct due to Lemma 3.

For sweeping the tangent rays of some given body $B_{i}, 1 \leq i \leq m$, the time requirements are dominated by the time to sort the tangent rays according to increasing angles. We can conclude:

THEOREM 4. Let the dimension be $d=2$. Then checking partial visibility of a body $B_{1}$ can be done with $O\left(\mathrm{~m}^{2} \log m\right)$ arithmetic steps, $O\left(\mathrm{~m}^{2}\right)$ calls to the first oracle, and $O\left(\mathrm{~m}^{2}\right)$ calls to the second oracle, as well as $O(m)$ space.

For other recent results on visibility computations in $\mathbb{R}^{2}$ see $[\mathbf{3}]$ and the references therein.

\section{Real enumerative geometry for the three-dimensional case}

In the three-dimensional case the basic algorithmic paradigm from Section 3 still holds. Since a line in $\mathbb{R}^{3}$ has four degrees of freedom, the core problem is now to compute the common tangent lines to four bodies in $\mathbb{R}^{3}$ (cf. $\left.[\mathbf{2 2}, \mathbf{1}]\right)$. However, in the three-dimensional case, there are also some special cases where we can transform a visibility ray only to a situation with 2 or 3 bodies, or where a configuration with four bodies has an infinite number of common tangents.

In particular, an algorithmic treatment of the latter situations (depending on the class $\mathcal{X}$ of bodies) does not only require to characterize the maximum possible number of tangents, but also asks for an a priori characterization of the configurations with infinitely many common tangents. In contrast to some other problems in computational geometry, characterizing these situations cannot be neglected (say, by applying perturbation techniques $[\mathbf{1 0}]$ ), since the large algebraic degree involved makes it usually highly nontrivial to guarantee a correct perturbation.

If the bodies are polytopes, the common tangents are common transversals of edges $[\mathbf{2 2}]$; so, in fact, the main geometric task is to compute the common transversals to four given lines in $\mathbb{R}^{3}$. This geometric problem has been well-known for many years (see, e.g., [13]). In particular, if a configuration has only finitely many common transversals, then this number is bounded by 2 ; and it is well-known how to characterize the configurations with infinitely many common transversals. 
However, the subsequent sections show that this situation completely changes if the set of bodies contains some non-linearly bounded bodies, such as balls.

4.1. Common tangent lines to four unit spheres. The following theorem in $[\mathbf{1 7}]$ characterizes the real algebraic-geometric situation when all bodies under investigation are unit balls.

Proposition 5. Four unit spheres in $\mathbb{R}^{3}$ have at most 12 common tangent lines unless their centers are located on the same line. Furthermore, there exists a configuration with 12 different real tangents, i.e., the upper bound is tight.

The proof of the upper bound provides a complex bound in the sense that it bounds the complex solutions of a system of polynomial equation. So, essentially, the theorem states that algebraically this tangent problem is of degree 12 . Note that due to this high degree, proving the characterization of the configurations with infinitely many common tangents is a highly nontrivial task.

From the viewpoint of projective geometry, this result is particularly interesting for the following reason. A line $\ell$ in three-dimensional projective space $\mathbb{P}^{3}$ can be represented in terms of its Plücker coordinates $p_{\ell} \in \mathbb{P}^{5}$ (see, e.g., $[\mathbf{1 3}, \mathbf{8}]$ ). In particular, it is well-known that the set of vectors in $\mathbb{P}^{5}$ satisfying the Plücker relation

$$
p_{01} p_{23}-p_{02} p_{13}+p_{03} p_{12}=0
$$

is in 1-1-correspondence with the set of lines in $\mathbb{P}^{3}$.

As described in the following, the condition that a line $\ell$ is tangent to a sphere gives a quadratic equation. Namely, for a given matrix $A \in \mathbb{R}^{m, n}$ we can use the wedge operator

$$
\wedge^{2}: \mathbb{R}^{m, n} \rightarrow \mathbb{R}^{\left(\begin{array}{c}
m \\
2
\end{array}\right),\left(\begin{array}{c}
n \\
2
\end{array}\right)}
$$

(cf. [29]). The row and column indices of the resulting matrix are subsets of cardinality 2 of $\{1, \ldots, m\}$ and $\{1, \ldots, n\}$, respectively. For $I \subset\{1, \ldots, m\}$ and $J \subset\{1, \ldots, n\}$ with $|I|=|J|=2$,

$$
\left(\wedge^{2} A\right)_{I, J}:=\operatorname{det} A_{[I, J]},
$$

where $A_{[I, J]}$ denotes the $2 \times 2$-submatrix of the matrix $A$ with row indices $I$ and column indices $J$. Describing a sphere with center $c \in \mathbb{R}^{3}$ and radius $r$ by the symmetric matrix

$$
Q=\left(\begin{array}{cccc}
c_{1}^{2}+c_{2}^{2}+c_{3}^{2}-r^{2} & -c_{1} & -c_{2} & -c_{3} \\
-c_{1} & 1 & 0 & 0 \\
-c_{2} & 0 & 1 & 0 \\
-c_{3} & 0 & 0 & 1
\end{array}\right)
$$

of the corresponding quadratic form $x^{T} Q x$, the following characterization of the tangent condition is applicable (see, e.g., $[\mathbf{2 9}]$ ).

LEMMA 6. A line $\ell \subset \mathbb{P}^{3}$ is tangent to a quadric $Q$ if and only if the Plücker vector $p_{\ell}$ of $\ell$ satisfies

$$
p_{\ell}^{T}\left(\wedge^{2} Q\right) p_{\ell}=0
$$

Hence, the set of lines tangent to four given unit spheres can be formulated by a system of five quadratic equations in $\mathbb{P}^{5}$. The reason why there are at most 12 solution in affine space instead of the (by Bézout's Theorem) expected $2^{5}=32$ solutions is that there is a one-dimensional component of solutions at infinity. It 
can be shown that this one-dimensional component of solutions accounts for the "missing" $2^{5}-12=20$ solutions. Quite remarkably, as observed in this intersectiontheoretical analysis [2], the excess component cannot be resolved by a single blowup.

Now let us have a look at the situation when the centers of the four spheres are affinely dependent. In $[\mathbf{1 8}]$ it is shown that under this condition the maximum number of real tangent lines goes down.

Proposition 7. If the four centers of four unit spheres in $\mathbb{R}^{3}$ are coplanar but not collinear, then there are at most 8 common tangent lines, and this bound is tight.

The proof of this statement uses some purely real algebraic arguments in the following sense. A line tangent to the four unit spheres can be seen as the axis of a unit cylinder whose surface passes through the four centers. Unless the axis is parallel to the plane of the four centers, its surface intersects this plane in an ellipse with semi-minor axis of length 1 . Each such ellipse corresponds to two cylinders. Using an appropriate algebraic description, it is shown in [17] that there can be at most 6 candidate conic sections satisfying the relevant algebraic properties, hence giving at most 12 common tangents. In addition to this (complex) algebraic argument, it is shown in [18] that some of the candidate conic sections are not ellipses but hyperbolas.

4.2. Realization issues. In order to find the common tangent lines we can either start from a system of polynomial equations, or we can construct a univariate polynomial equation whose solutions encode the tangents. For both approaches the numerical computation of the tangents may become instable, especially for configurations of centers which are close to singular configurations (e.g., configurations corresponding to reducible polynomials in an algebraic description). Hence, it is essential to treat realization questions, such as: For which numbers $k \in\{0, \ldots, 12\}$ do there exist configurations with exactly $k$ different common tangents in real space?

If not all numbers $k \in\{0, \ldots, 12\}$ can be established in real space this offers the possibility of strong and valuable consistency checks within a program. If, however, all numbers can be realized then this would prove the non-existence of such a control mechanism. Indeed, the latter result is true, as stated in the following theorem in $[31]$.

THEOREM 8. For any number $k \in\{0, \ldots, 12\}$ there exists a configuration of 4 unit spheres in $\mathbb{R}^{3}$ which have exactly $k$ different common tangents in $\mathbb{R}^{3}$.

This result contrasts the 4-sphere-problem to some well-studied problems in classical and enumerative geometry. For example, concerning one of the most famous problems from enumerative geometry, the number of 27 lines on a smooth cubic surface, the question of real solutions has already been studied long time ago $\left([\mathbf{2 5}, \mathbf{2 7}]\right.$, see also [24], p. 188). In particular, for a cubic surface in $\mathbb{R}^{3}$ only the numbers 3, 7, 15, and 27 can be established with real lines. Another famous example in geometry is Apollonius' problem which asks for the circles tangent to three given circles. For this problem, there exist configurations with $k \in\{0,1, \ldots, 6,8\}$ real tangent circles but provably no configuration with 7 real tangent circles $[\mathbf{2 1}]$. 


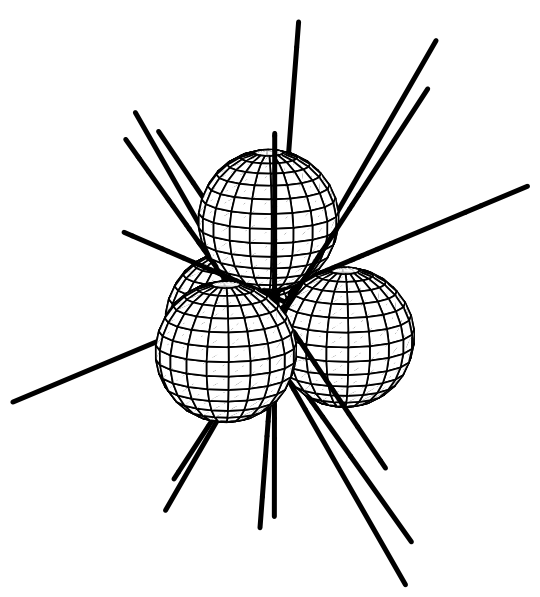

Figure 5. Construction of four unit spheres with nine common tangent lines

As an example of the geometry of the constructions, Figure 5 shows a configuration with four unit spheres and nine common tangent lines. For configurations with twelve common tangent lines see $[\mathbf{1 7}, \mathbf{3 1}]$.

4.3. Combinations of spheres and polytopes. The next question is, what happens when the class of admissible bodies in the scene consists of both balls and polytopes. From the algebraic-geometric viewpoint, this immediately leads to the problem of finding the common tangents/transversals to $k$ spheres and $n-k$ lines, $k \in\{0, \ldots, 4\}$. For convenience of notation, we consider a transversal of a line as a tangent to the line.

Tight upper bounds for the number of common tangents to $k$ spheres and $n-k$ lines are known in the finite case, $k \in\{0, \ldots, 4\}$. Here, as in Section 4.1, tightness refers to the following (quite strong) sense of real algebraic geometry (cf. [30]): On the one hand, for each $k$ we bound the number of solutions by algebraic methods, say, by some number $m$. Then, on the other hand, constructions are known which indeed leads to $m$ solutions in real space $\mathbb{R}^{3}$ (which would not be possible if, say, any polynomial formulation contained some complex solutions).

The general difficulty of proving tight bounds of this kind may be seen by the following two aspects. For the classical enumerative geometry problem of conics tangent to five given conics (dating back to Steiner in 1848) the existence problem of 3264 real solutions had not been solved until few years ago ([23] and [12], $\S 7.2)$. Furthermore, as pointed out in [30] (cf. also [28]), there are nearly no criteria or general techniques for tackling questions of this type. For these reasons, it is even more remarkable that in all of the situations there exists a construction matching the upper bound.

Table 4.3 summarizes the results from [32]. It shows the upper bounds for the number of solutions and the matching numbers of real solutions in the best constructions. The last column shows that in only a few cases, explicit characterizations of the configurations with an infinite number of common tangents are known so far. Namely, besides the already mentioned results for 4 lines and for 


\begin{tabular}{|l||r|r|c|}
\hline \hline & $\begin{array}{r}\text { upper bound } \\
\text { \# solutions }\end{array}$ & $\begin{array}{r}\text { \# real solutions of } \\
\text { a construction }\end{array}$ & $\begin{array}{c}\text { characterization of } \\
\text { degenerate instances }\end{array}$ \\
\hline \hline 4 lines & 2 & 2 & yes \\
3 lines, 1 sphere & 4 & 4 & yes \\
2 lines, 2 spheres & 8 & 8 & - \\
1 line, 3 spheres & 12 & 12 & - \\
4 unit spheres & 12 & 12 & yes \\
4 spheres & 12 & 12 & - \\
\hline \hline
\end{tabular}

TABLE 1. Summary of results

4 unit spheres, this characterization is only known for the case of three lines and one sphere. In entries with a "-_" such a characterization is not known yet.

Let $N_{k}$ denote the maximum number of common tangents/transversals to $k$ spheres and $4-k$ lines in the finite case, $0 \leq k \leq 4$. The proofs for the tight upper bounds of $N_{0}, \ldots, N_{4}$ in [32] are of quite different flavors. For $k \in\{1,2\}$, the upper bounds immediately follow from Bézout's Theorem. Whereas for $k=1$ it is easy to give a construction matching this bound, the correctness proof of the construction for $k=2$ is based on the computer-algebraic technique of using standard bases. For $N_{3}=12$, the Bézout bound of a projective formulation is 16 instead of 12 . In order to prove the bound of 12 , by analyzing the underlying algebraic geometry of the problem, two double solutions at infinity can be spotted. Finally, for the proof for $N_{4}$ (for general radii) there are two possibilities. An appropriate elementary geometric description can be used to deduce a polynomial formulation with Bézout bound 12, or one can use that the intersection theory technique mentioned in Section 4.1 also holds for general radii.

\section{On the smallest radius giving common tangent lines}

In the previous section we dealt with the enumerative geometry problem to count, characterize, and find the common transversals/tangent lines to given bodies. In this section we would like to illustrate another relationship between these problems and real algebraic geometry: that of optimizing polynomial functions. This aspect is not only relevant for optimization aspects with regard to the visibility/tangent problems, but in particular is also related to the problem of finding smallest enclosing/circumscribing cylinders of arbitrary point sets in $\mathbb{R}^{3}$ (cf. $[\mathbf{1}, \mathbf{7}, \mathbf{2 6}])$.

Let $c_{1}, \ldots, c_{4}$ be affinely independent. Then, of course, there does not exist a line passing through all of these points. However, for every edge of the tetrahedron $c_{1} c_{2} c_{3} c_{4}$, there exists a line which is parallel to that edge and which is tangent to the four spheres $S\left(c_{i}, r\right)$ for some radius $r>0$ (see [17]). Hence, there exists a smallest radius $r>0$ such that the four spheres $S\left(c_{i}, r\right)$ have a common tangent line. A tangent line for this radius $r$ is called a tangent line with minimal radius. In general, this problem is a polynomial optimization problem over the real numbers, and - by the results in Section 4 on the algebraic degree involved - it is hard to find the exact global optima (cf. the discussion in $[\mathbf{2 0}]$ ). In particular, the latter paper deals with new ideas to find global optima of certain unconstrained real polynomial optimization problems. Generally, we think that for geometric problems like finding 
the tangent line of minimal radius it is an important and challenging question to combine general methods for polynomial optimization (like the one mentioned) with specific geometric insights.

To provide an example for combinations of this kind, let us again look at the 4-sphere-problem. Of course, it is hard to find good geometric characterizations in the general case. However, for an interesting subclass of 4-sphere-configurations, we can prove the following geometric characterization.

THeOREM 9. Let $c_{1} c_{2} c_{3} c_{4}$ define an equifacial tetrahedron, and let $\ell$ be a tangent line to $S\left(c_{1}, r\right), \ldots, S\left(c_{4}, r\right)$ with minimal radius $r$. Then the direction vector of $\ell$ is perpendicular to the direction vectors of two opposite edges, and each of these two edges has length $2 r$.

In order to prove this theorem, the following notation of [17] is used. Let $c_{1}, \ldots, c_{4}$ be affinely independent. Then the four centers define a tetrahedron $T$ in $\mathbb{R}^{3}$. Further, let $\ell=\{p+\mu s: \mu \in \mathbb{R}\}$ with $p, s \in \mathbb{R}^{3}, s \neq 0, p \perp s$, be a line. $\ell$ is tangent to the four spheres $S\left(c_{i}, r\right)$ with radius $r>0$ if the line $\ell-p$ (which passes through the origin) has distance $r$ from the four points $c_{i}-p$, i.e, if and only if

$$
\left(\left(c_{i}-p\right) \times s\right)^{2}=r^{2} s^{2}, \quad 1 \leq i \leq 4 .
$$

PROOF. It is well-known that the vertices of an equifacial tetrahedron $T$ can be regarded as four pairwise non-adjacent vertices of a rectangular box (see, e.g., [14]). Hence, there exists a representation $c_{1}=\left(\lambda_{1}, \lambda_{2}, \lambda_{3}\right)^{T}, c_{2}=\left(\lambda_{1},-\lambda_{2},-\lambda_{3}\right)^{T}$, $c_{3}=\left(-\lambda_{1}, \lambda_{2},-\lambda_{3}\right)^{T}, c_{4}=\left(-\lambda_{1},-\lambda_{2}, \lambda_{3}\right)^{T}$ with $\lambda_{1}, \lambda_{2}, \lambda_{3}>0$.

Assuming without loss of generality $s^{2}=1$, application of Lagrange's identity on (2) gives

$$
\left(\left(c_{i}-p\right) \times s\right)^{2}=c_{i}^{2}-\left\langle c_{i}, s\right\rangle^{2}-2\left\langle c_{i}, p\right\rangle+p^{2} .
$$

Hence, Equation (2) takes the form

$$
\left\langle c_{i}, s\right\rangle^{2}+2\left\langle c_{i}, p\right\rangle=\sum_{j=1}^{3} \lambda_{j}^{2}+p^{2}-r^{2}, \quad 1 \leq i \leq 4 .
$$

Subtracting these equations pairwise gives

$$
4\left(\lambda_{2} p_{2}+\lambda_{3} p_{3}\right)=-4\left(\lambda_{1} \lambda_{3} s_{1} s_{3}+\lambda_{1} \lambda_{2} s_{1} s_{2}\right)
$$

(for indices 1,2) and analogous equations, so that

$$
\lambda_{1} p_{1}=-\lambda_{2} \lambda_{3} s_{2} s_{3}, \quad \lambda_{2} p_{2}=-\lambda_{1} \lambda_{3} s_{1} s_{3}, \quad \lambda_{3} p_{3}=-\lambda_{1} \lambda_{2} s_{1} s_{2} .
$$

Since $\langle p, s\rangle=0$, this yields $s_{1} s_{2} s_{3}=0$. Without loss of generality we can assume $s_{1}=0$. In this case,

$$
p=\left(-\frac{\lambda_{2} \lambda_{3}}{\lambda_{1}} s_{2} s_{3}, 0,0\right)^{T} .
$$

So we can express (3) in terms of the direction vector $s$,

$$
\lambda_{2}^{2} s_{2}^{2}+\lambda_{3}^{2} s_{3}^{2}=\sum_{j=1}^{3} \lambda_{j}^{2}+\left(-\frac{\lambda_{2} \lambda_{3}}{\lambda_{1}} s_{2} s_{3}\right)^{2}-r^{2},
$$

which, by using $s_{2}^{2}+s_{3}^{2}=1$, gives

$$
\lambda_{2}^{2} \lambda_{3}^{2} s_{2}^{4}+\left(\lambda_{1}^{2} \lambda_{2}^{2}-\lambda_{1}^{2} \lambda_{3}^{2}-\lambda_{2}^{2} \lambda_{3}^{2}\right) s_{2}^{2}+\lambda_{1}^{2}\left(r^{2}-\lambda_{1}^{2}-\lambda_{2}^{2}\right)=0
$$


In particular, $s=(0,1,0)^{T}$ gives $r^{2}=\lambda_{1}^{2}+\lambda_{3}^{2}$, and $s=(0,0,1)^{T}$ gives $r^{2}=\lambda_{1}^{2}+\lambda_{2}^{2}$. By setting $z_{2}=s_{2}^{2}$, Equation (4) defines a quadratic function $r^{2}\left(z_{2}\right)$,

$$
r^{2}\left(z_{2}\right)=\frac{1}{\lambda_{1}^{2}}\left(-\lambda_{2}^{2} \lambda_{3}^{2} z_{2}^{2}-\left(\lambda_{1}^{2} \lambda_{2}^{2}-\lambda_{1}^{2} \lambda_{3}^{2}-\lambda_{2}^{2} \lambda_{3}^{2}\right) z_{2}\right)+\lambda_{1}^{2}+\lambda_{2}^{2} .
$$

Since this function is concave in $z_{2} \in[0,1]$, one of the given boundary values of $r^{2}$ is the minimum value. Moreover, since both $2 \sqrt{\lambda_{1}^{2}+\lambda_{2}^{2}}$ and $2 \sqrt{\lambda_{1}^{2}+\lambda_{3}^{2}}$ are the lengths of a pair of opposite edges, the statement follows.

Acknowledgments. Thanks to Abhi Dattasharma for his helpful comments.

\section{References}

[1] P.K. Agarwal, B. Aronov, and M Sharir. Line transversals of balls and smallest enclosing cylinders in three dimensions. Discrete Comput. Geom., 21:373-388, 1999.

[2] P. Aluffi and W. Fulton. Personal communication.

[3] P. Angelier and M. Pocchiola. A sum of squares theorem for visibility complexes. In Proc. Symposium on Computational Geometry (Medford, MA), 2001.

[4] M. Bern, D. Dobkin, D. Eppstein, and R. Grossman. Visibility with a moving point of view. Algorithmica, 11:360-378, 1994.

[5] T. Bonnesen and W. Fenchel. Theorie der konvexen Körper. Springer-Verlag, 1934. English translation: BCS Associates, Moscow, Idaho, 1987.

[6] S.E. Cappell, J.E. Goodman, J. Pach, R. Pollack, M. Sharir, and R. Wenger. Common tangents and common transversals. Adv. Math., 106:198-215, 1994.

[7] T.M. Chan. Approximating the diameter, smallest enclosing cylinder, and minimum-width annulus. In Proc. Symposium on Computational Geometry (Clear Water Bay, Hong Kong), pages 300-309, 2000.

[8] D. Cox, J. Little, and D. O'Shea. Ideals, Varieties, and Algorithms. Springer-Verlag, New York, 2nd edition, 1996

[9] M. de Berg, M. van Kreveld, M. Overmars, and O. Schwarzkopf. Computational Geometry. Springer-Verlag, Berlin, 2nd edition, 2000.

[10] H. Edelsbrunner and E.P. Mücke. Simulation of simplicity: A technique to cope with degeneracy. ACM Trans. Graph., 9(1):43-72, 1990.

[11] H. Edelsbrunner and M. Sharir. The maximum number of ways to stab $n$ convex nonintersecting sets in the plane is $2 n-2$. Discrete Comput. Geom., 5:35-42, 1990.

[12] W. Fulton. Introduction to Intersection Theory in Algebraic Geometry. CBMS 54. AMS, 2nd edition, 1996.

[13] W. Hodge and D. Pedoe. Methods of Algebraic Geometry, volume 2. Cambridge University Press, 1952.

[14] Y.S. Kupitz and H. Martini. Equifacial tetrahedra and a famous location problem. Math. Gazette, 83:464-467, 1999.

[15] H.-P. Lenhof and M. Smid. Maintaining the visibility map of spheres while moving the viewpoint on a circle at infinity. Algorithmica, 13:301-312, 1995.

[16] T. Lewis, B. von Hohenbalken, and V. Klee. Common supports as fixed points. Geom. Dedicata, 60:277-281, 1996.

[17] I.G. Macdonald, J. Pach, and T. Theobald. Common tangents to four unit balls in $\mathbb{R}^{3}$. Discrete Comput. Geom., 26:1-17, 2001.

[18] G. Megyesi. Lines tangent to 4 spheres with affinely dependent centres. Discrete Comput. Geom., 26:493-497, 2001.

[19] J. O'Rourke. Visibility. In J.E. Goodman and J. O'Rourke, editors, Handbook of Discrete and Computational Geometry, pages 467-479. CRC Press, Boca Raton/NY (USA), 1997.

[20] P.A. Parrilo and B. Sturmfels. Minimizing polynomial functions. This volume.

[21] D. Pedoe. The missing seventh circle. Elem. Math., 25:14-15, 1970.

[22] M. Pellegrini and P.W. Shor. Finding stabbing lines in 3-space. Discrete Comput. Geom., pages 191-208, 1992. 
[23] F. Ronga, A. Tognoli, and T. Vust. The number of conics tangent to 5 given conics: the real case. Rev. Mat. Univ. Complut. Madrid, 10:391-421, 1997.

[24] G. Salmon. A Treatise on the Analytic Geometry of Three Dimensions, volume 1. Longmans and Green, London, 7th edition, 1928. Reprinted by Chelsea Publishing Company, New York, 1958.

[25] L. Schläfli. On the distributions of surfaces of the third order into species, in reference to the absence or presence of singular points, and the reality of their lines. Phil. Trans. Royal Society of London, 153:193-241, 1863.

[26] E. Schömer, J. Sellen, M. Teichmann, and C. Yap. Smallest enclosing cylinders. Algorithmica, 27:170-186, 2000.

[27] B. Segre. The Non-Singular Cubic Surfaces. Oxford University Press, 1942.

[28] F. Sottile. Enumerative real algebraic geometry. This volume.

[29] F. Sottile. From enumerative geometry to solving systems of polynomial equations with Macaulay 2. In Computations in Algebraic Geometry with Macaulay 2, ed. by D. Eisenbud, D. Grayson, M. Stillman, and B. Sturmfels. Springer-Verlag, Berlin, 2001.

[30] F. Sottile. Enumerative geometry for the real Grassmannian of lines in projective space. Duke Math. J., 87:59-85, 1997.

[31] T. Theobald. How to realize a given number of tangents to four unit balls in $\mathbb{R}^{3}$. To appear in Mathematika.

[32] T. Theobald. An enumerative geometry framework for algorithmic line problems in $\mathbb{R}^{3}$. To appear in SIAM J. Computing.

[33] C.A. Wang and B. Zhu. Three-dimensional weak visibility: Complexity and applications. Theor. Comp. Sci., 234:219-232, 2000.

[34] J. Wernecke. The Inventor Mentor: Programming Object-Oriented 3D Graphics with Open Inventor. Addison-Wesley, Reading, MA, 1994.

Thorsten Theobald, Zentrum Mathematik, Technische Universität München, D80290 München, Germany

E-mail address: theobald@ma.tum.de 